\title{
P128: Improving hand hygiene compliance in a teaching hospital
}

\author{
R Cocconi ${ }^{*}$, L Arnoldo ${ }^{2}$, M Dal Cin², P Del Giudice ${ }^{2}$, R Fabro ${ }^{1}$, A Faruzzo ${ }^{1}$, D Tignonsini' ${ }^{1}$ S Brusaferro ${ }^{2}$ \\ From 2nd International Conference on Prevention and Infection Control (ICPIC 2013) \\ Geneva, Switzerland. 25-28 June 2013
}

\section{Introduction}

Although WHO project "Clean care is safer care" was introduced many years ago in our hospital, Hand Hygiene ( $\mathrm{HH})$ practice shows low compliance level in some wards at increased risk of Healthcare Associated Infections (HAI).

\section{Objectives}

The project objective is to determine the causes of $\mathrm{HH}$ non-compliance and to identify targeted solutions for improvement.

\section{Methods}

The project was carried out with the collaboration of Joint Commission Center for Transforming Healthcare, using TST@ methodology, from April to September 2012. We chose to implement the project in a pilot ward, an internal medicine ward.

We selected two groups of observers. The first ones, the secret observers, had the mandate to see whether the individual washed his or her hands upon entering and exiting the room. The second group had trained to be just-in-time (JIT) coaches. The JIT coaches approached health care workers who are found to not be washing hands appropriately and query them as to the reasons for their non-compliance (non observable contributing factors).

\section{Results}

After 3 weeks of data collection experience, with secret observers, the baseline $\mathrm{HH}$ compliance in the pilot unit was an average of $26.7 \%$. Entering room was worse than exit room, $22.4 \%$ versus $31,7 \%$.
Non-compliance contributing factors were: improper use of gloves (57.3\%), frequently entry or exit room (15.5\%), hands full of supplies $(7.8 \%)$, follow person in exit or entry room (6.8\%) and equipment shared (5.8\%).

Non observable contributing factors, coming from JIT coaches, were: perception that $\mathrm{HH}$ is not necessary (50.0\%), distracted (41.7\%) and skin irritation (8.3\%).

After one month of targeted solution implemented, such us: relocation of glove dispensers in the ward, standardize work processes, use a code word for distracted people, etc. $\mathrm{HH}$ compliance rose to an average of $65 \%$ with a gain of $34,7 \%$.

\section{Disclosure of interest}

None declared.

\section{Author details}

'Direzione Medica di Presidio, Azienda Ospedaliero-Universitaria Santa Maria della Misericordia, Italy. ${ }^{2}$ Department of Medical and Biological Sciences, University of Udine, UDINE, Italy.

Published: 20 June 2013

\section{doi:10.1186/2047-2994-2-S1-P128}

Cite this article as: Cocconi et al.: P128: Improving hand hygiene compliance in a teaching hospital. Antimicrobial Resistance and Infection Control 2013 2(Suppl 1):P128. 